\title{
Semi-Automatic Tools to Segment Sem Images of Particles
}

\author{
D. S. Bright
}

Surface and Microanalysis Science Division, National Institite of Standards and Technology, Gaithersburg, MD. 20899-8371

Shaded SEM images of particles on textured backgrounds are difficult to segment using traditional means, and tedious to segment by manually tracing the boundary. Characterization of particles in digital images requires first that the images be segmented, that is, that the particles be separated from the background. This paper describes rules-of-thumb and public domain digital image processing tools[1] that run under Windows* for segmenting these images. The images are flattened to remove the shadows without distorting the edges, and then breaks in the boundary are patched manually. Given a proper particle boundary, image analysis can proceed: the particle projected area is a count of its pixels, the maximum diameter is the distance between the two pixels in the particle that are farthest apart, etc.

SEM images are often taken so that the particle is shaded because these images are more pleasing and give a better feel for particle shape. Shadows on the particle make parts of its surface darker than parts of the background - a single intensity threshold will not work (Fig. 1a). To make the over-all brightness of the image more uniform, a 'background' image is made by smoothing the image with a mean (averaging) filter, using a kernel edge of 5 to $20 \%$ of the particle diameter. This obliterates fine detail such as the particle edges, and retains the shading. When this background image is subtracted from the original, the edges of the particle remain, and the background is a more or less uniform shade of gray (Fig. 1b). This difference or flattened image lacks visual appeal, and may no longer clearly represent the three-dimensional morphology of the particle, but this image can be thresholded such as to select the edge of the particle, or most of it. Commonly, the edge will have gaps, often small, which allow the resulting boundary to leak into the interior of the particle or out into the boundary (Fig. 1c). To handle situations like these, I made manual tools, called 'Patch', 'Split', and 'Join', which require a minimum of manual intervention. They use mouse clicks to select points where the boundary diverts from where it should go (circles, Fig. 1c). The tools connect the points to obtain the intact particle outline (Fig. 1d).

The boundary editing tools work as follows: Patch connects two boundary points (Fig. 2c) with a straight (or optionally, a manually drawn) line, separating the boundary into two pieces (Figs. 2d,e), one of which the user selects. This is for eliminating leaks into the interior or background, or tendrils that are obviously not part of the particle. Split is the same as "Patch", except that both pieces are kept as separate blobs (Fig. 2g) - this is useful for separating touching particles. Join connects two pairs of boundary points with straight lines, thus connecting two blobs together (Fig. Ұ,k,1).

I tested these tools by segmenting a collection of 1500 SEM images of shaded particles. This took about a week, working on the images half time. Some images took 20 seconds to segment, a few, which had partially obscured, indiscernible boundaries took about ten minutes. Most images took one or two minutes to segment.

Although this segmentation method is not automatic, it works on a variety of images, which is not the case for automatic methods. I found that many times, segmentation required a judgment of boundary location which an automatic method might not make properly. The time to segment images manually was not much more than the time to check automatically segmented boundaries of other image sets that were not so drastically shaded or had darker, less textured backgrounds. 
References:

[1] Tools available for download atiwww.nist.gov/lispix.

* Certain commercial equipment, instruments, or materials are identified in this report to specify adequately the experimental procedure. Such identification does not imply recommendation or endorsement by the National Institute of Standards and Technology, nor does it imply that the materials or equipment identified are necessarily the best available for the purpose.
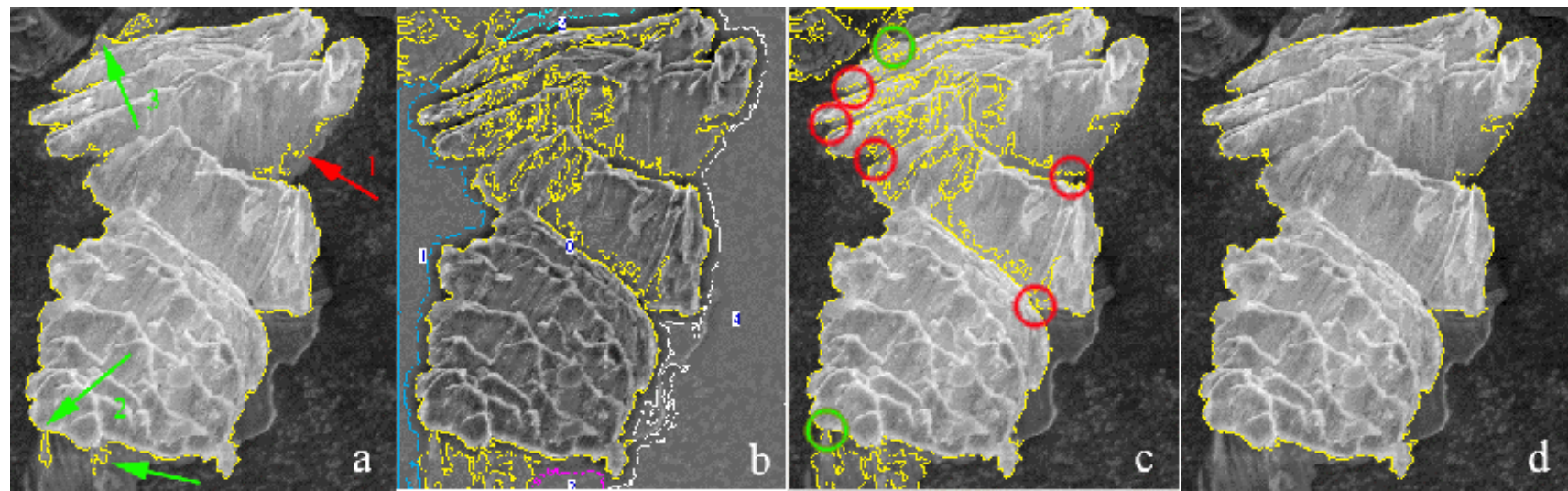

FIG. 1 Segmentation of SEM image of $100 \mu \mathrm{m}$ brass filing. a) Original, threshold gives boundary (yellow) missing darker area (arrow 1), but including some background (arrows 2,3). b) Flattened image, threshold gives four blobs: blob 0 follows boundary, but also wanders around. Other blobs discarded. c) Boundary from b superimposed on particle in a. Red and green circles - locations for 'patching' (see below) to omit interior and exterior 'leaks' respectively. d) Completed boundary.
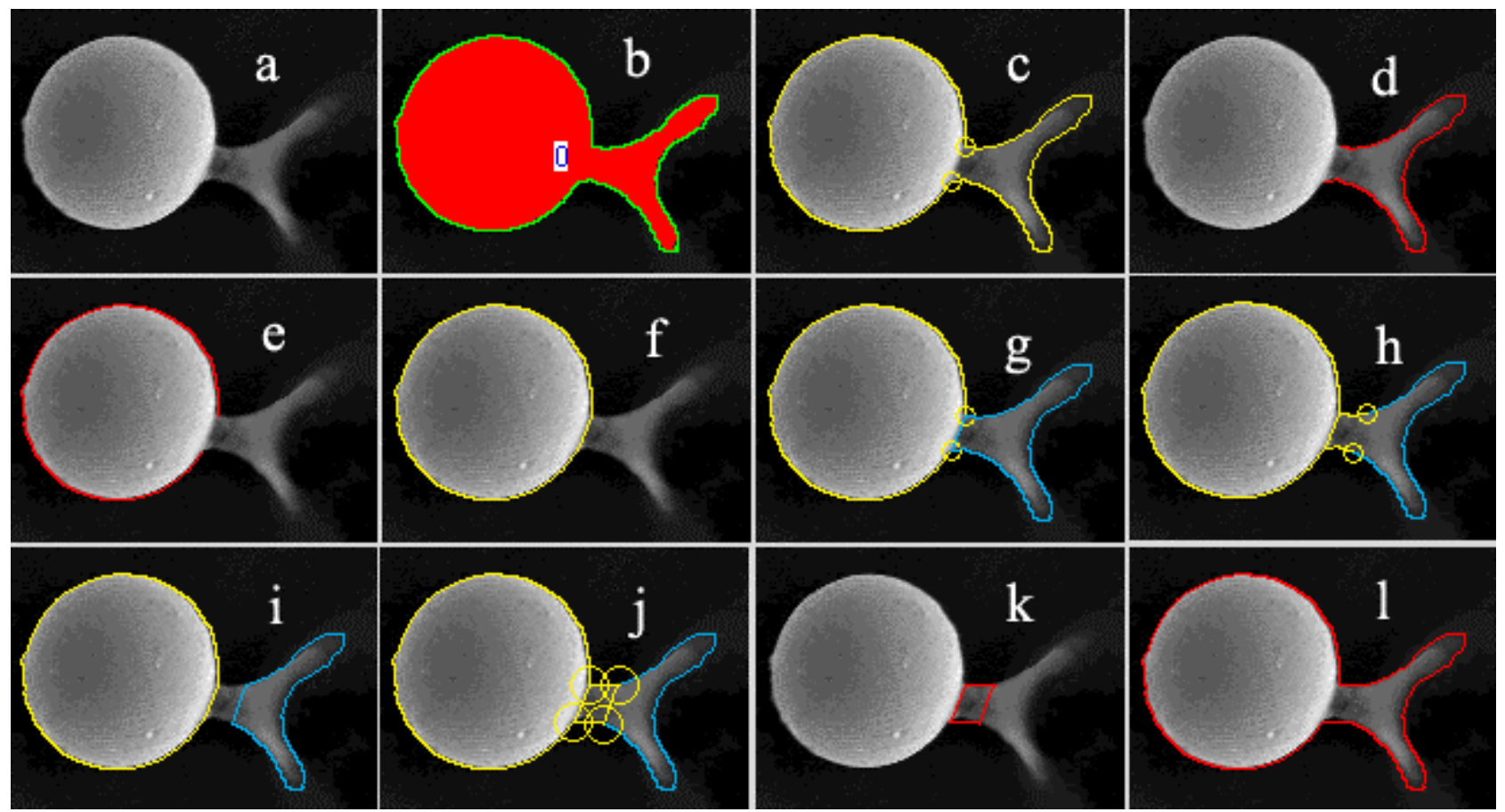

FIG. 2. Illustration of patch, split and join tools. a. $50 \mu \mathrm{m}$ sphere, tail of goop. b. Threshold (red), outline (green). c. Clicking selects two points (yellow circles), dividing boundary into two parts. d. One part selected. e. Other part selected. f. 'Patch' completes boundary in e. g. Split, after step c, retains both parts in $\mathrm{d}$ and e. h,i. Blue blob shortened using 'patch. j. Four points selected. $\mathrm{k}$. Wrong point-edge combination. 1. Correct edge combination after join. 